\title{
Strangers in the Night-Smart Process Sensors in Our Current Automation Landscape ${ }^{\dagger}$
}

\author{
Michael Maiwald *, Patrick Gräßer, Lukas Wander, Nicolai Zientek, Svetlana Guhl, \\ Klas Meyer and Simon Kern \\ Bundesanstalt für Materialforschung und -prüfung (BAM), 12489 Berlin, Germany; \\ patrick.graesse@gmx.de (P.G.); lukas.wander@bam.de (L.W.); nicolai.zientek@googlemail.com (N.Z.); \\ svetlana.guhl@bam.de (S.G.); klas.meyer@bam.de (K.M.); simon.kern@bam.de (S.K.) \\ * Correspondence: michael.maiwald@bam.de; Tel.: +49-30-8104-1140 \\ + Presented at the Eurosensors 2017 Conference, Paris, France, 3-6 September 2017.
}

Published: 21 August 2017

\begin{abstract}
The departure from the current automation landscape to next generation automation concepts for the process industry has already begun. Smart functions of sensors simplify their use and enable plug-and-play integration, even though they may appear to be more complex at first sight. Smart sensors enable concepts like self-diagnostics, self-calibration, and self-configuration/parameterization whenever our current automation landscape allows it. Here we summarize the currently discussed general requirements for process sensors 4.0 and introduce a smart online NMR sensor module as example, which was developed for an intensified industrial process funded by the EU's Horizon 2020 research and innovation programme (www.consens-spire.eu).
\end{abstract}

Keywords: smart sensors; intensified processes; sustainable production; reaction monitoring; NMR spectroscopy; Industry 4.0; Industrial Internet of Things (IIoT); continuous manufacturing

\section{Introduction}

The future competitiveness of the process industry and their providers depends on its ability to deliver high quality and high value products at competitive prices in a sustainable fashion, and to adapt quickly to changing customer needs. Competitiveness therefore means safeguarding of the required product quality with an optimum use of equipment, raw materials, and energy.

Process analytical techniques are extremely useful tools for chemical production and manu-facture particularly for the pharmaceutical, food, and (petro-) chemical industries. It can be easily transferred to manufacturing for process control and for quality assurance of final products to meet required product specifications, since it provides dynamic information about product properties, material stream characteristics, and process conditions [1].

\section{Process Control Based on Intelligent Field Devices}

Intelligent field devices, digital field networks, Internet Protocol (IP)-enabled connectivity and web services, historians, and advanced data analysis software are providing the basis for the future project "Industrie $4.0^{\prime \prime}$ and Industrial Internet of Things (IIoT). This is a prerequisite for the realization of Cyber-Physical Systems (CPS) within these future automation concepts for the process industry. "Cyber-Physical Systems are integrations of computation with physical processes. Embedded computers and networks monitor and control the physical processes, usually with feedback loops where physical processes affect computations and vice versa" [2]. 


\subsection{Integrated Process Design and Intensified Processes - Need for Smart Field Devices}

Novel discoveries, developments, and concepts in the field of process engineering and in particular process intensification are currently promoted for analysis and design of innovative equipment and processing methods. This leads to substantially improved sustainability, efficiency, and environmental performance, alternative energy conversion, alternative transport mechanisms, intensified hydrodynamics, structured environments, multi functionality, and intensified plant operation.

Intensified continuous processes are in focus of current research. Compared to traditional batch processes, intensified continuous production gives admittance to new and difficult to produce compounds (see Section 3. as an example), leads to better product uniformity, and drastically reduces the consumption of raw materials and energy. Flexible (modular) chemical plants can produce various products using the same equipment with short down-times between campaigns, and quick introduction of new products to the market. Typically, such plants have smaller scale than plants for basic chemicals in batch production but still are capable to produce kilograms to tons of specialty products each day.

Consequently, full automation is a prerequisite to realize such benefits of intensified continuous plants. In continuous flow processes, continuous, automated measurements and tight closed-loop control of the product quality are mandatory. If these are not available, there is a huge risk of producing large amounts of out-of-spec (OOS) products.

In pharmaceutical production, the common future vision is "continuous manufacturing" (CM), based on "Real Time Release" (RTR), i.e., a risk based and integrated quality control in each process unit. This will allow for flexible hook-up of smaller production facilities, production transfer towards fully automated facilities, less operator intervention, less down time, and end to end process understanding over product lifecycle, future knowledge, and faster product to market. It is also assumed to significantly reduce the quality control costs within a CM concept at the same time.

\subsection{Lack of Contemporary Process Control Concepts in Our Current Automation Landscapes}

Current focuses of research are closed-loop adaptive control concepts for plant-wide process control, which make use of specific or non-specific sensors along with conventional plant instruments. Such advanced control solutions could give more information than only control information, such as sensor failure detection, control performance monitoring, and improve simulation-based engineering as one of the concepts in Industry 4.0 or IIoT.

With the introduction of advanced process analytical technology, the closeness of key process variables to their limits can be directly monitored and controlled and the processes can automatically be driven much closer to the optimal operating limit. Classical, non-model-based solutions reach their limits when sensor information from several sources must be merged. In addition, their adaptation causes a high effort during the life cycle of the process. This highlights the need for adaptive control strategies, which are based on dynamic process models as mentioned above. Model-based control concepts have also the potential to automatically cope with silent changes of the raw-materials as well as process conditions.

Chemical process control technology has advanced significantly during the last decades. For world-scale high-throughput continuous processing units such as crackers and separation trains, in most cases classically engineered control solutions have been replaced by model-based techniques, most prominently model-predictive control (MPC) based on linear plant models. However, the engineering and implementation costs of such advanced controllers are still high. For smaller, flexible processes in which varying products or intermediates are manufactured, it is not economic to re-engineer the control concept or to re-model the process for all intended processes-especially for a modular approach.

Chemometric techniques for the derivation of empirical models (e.g., PLS (Partial Least Squares), PCA (Principal Component Analysis)) are available but currently mostly used for off-line data analysis to detect the causes of variations in the product quality. An automated application along the life cycle is still very limited. Up to now the development of such models requires significant experimental work. The use of first principles methods (e.g., spectroscopic models, cf. Section 3.) 
along with reduction of the effort needed for these experiments is focus of ongoing research. When such stationary models are available and are combined with dynamic models that describe the times needed for the transition from one steady state to the other, feedback control and iterative optimization schemes can be built. Making use of novel sensors, like online NMR, tremendously promote the use of novel process control concepts.

\subsection{Current and Future Requirements to Process Sensors and Actuators}

Sensors are the sense organs of process automation. At present, there are serious changes in the areas of information and communication technology, which offer a great opportunity for optimized process control and value added production with dedicated network communicating sensors. These kinds of "smart" sensors provide services within a network and use information from it. Consequently, smart process sensors enable new business models for users, device manufacturers, and service providers.

Recently the technology roadmap "Process Sensors 4.0" was published [3], which identifies the necessary requirements as well as the communication abilities of such process sensors-from a simple temperature sensor up to current technologies, which are in development. Important smart features are depicted in Figure 1.

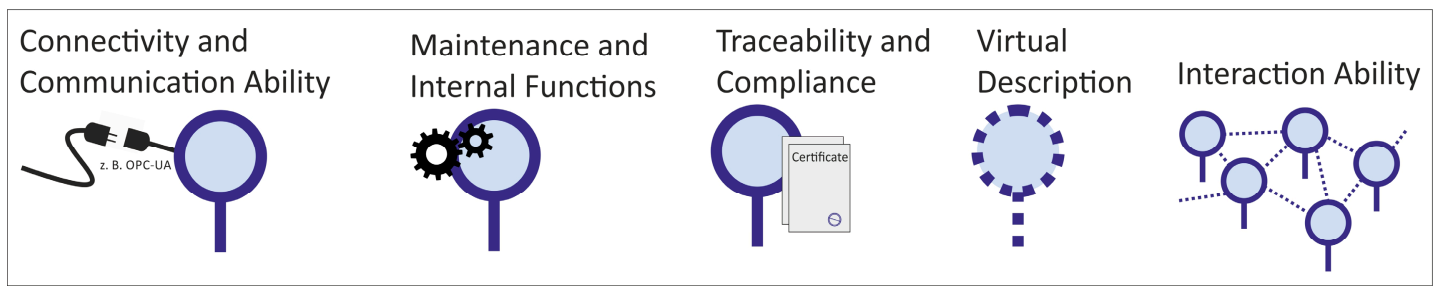

Figure 1. Most important features of a smart sensor or actuator [3].

The cost of connectivity is dropping dramatically, providing powerful potential to connect people, assets, and information across the industrial enterprise. While only providing add-on information, the first cloud services may not require a high disposability or real-time capabilities. But are these given in future, even process control tasks were possible using cloud services, e.g., when complex computing algorithms are needed, which require computing power.

\section{3. "Chemistry for Chemistry"-Smart Compact NMR Spectroscopy in Process Control}

Monitoring specific information (i.e., "chemical" such as physico-chemical properties, chemical reactions, etc.) is the key to "chemical" process control. As an example, a given pharmaceutical aromatic coupling reaction step (lithiation reaction) was investigated (Figure 2a). [4] The challenge within the project was to integrate a commercially available low-field NMR spectrometer from a laboratory application to the full requirements of an automated chemical production environment including robust evaluation of sensor data.

Today, mainly optical online methods are applied. Quantitative online nuclear magnetic resonance (NMR) spectroscopy has a high potential for direct loop process control of fluid process streams $[1,3]$ since it provides rapid and non-invasive information when applied in a flow through mode. One of the most attractive features of quantitative NMR spectroscopy is that NMR peak areas can be directly used for concentration quantification without further calibration-if complete spin magnetisation is achieved. A major advantage of NMR spectroscopy is that the method features a high linearity between absolute signal area and sample concentration, which makes it an absolute analytical comparison method which is independent of the matrix. This is an important prerequisite for robust data evaluation strategies within a control concept and reduces the need for extensive maintenance of the evaluation model over the time of operation.

Compact NMR instruments based on permanent magnets are robust and relatively inexpensive analysers. [1] The developed smart NMR sensor is provided in an explosion proof housing (Figure 2b) involves a compact $43.5 \mathrm{MHz}$ NMR spectrometer together with an acquisition unit and a 
programmable logic controller for fully automated data preparation (phasing, baseline correction) and quantitative evaluation. Therefore, a spectral modeling procedure based on Indirect Hard Modeling (IHM) was developed and validated for data analysis of the low-field NMR spectra. Such spectral models can be built assigning all pure components within a few hours from first principles information (i.e., assignment of the NMR signals to the components) instead of tedious calibrations runs based on, e.g., a Design of Experiments (DoE).

In addition to the NMR information further controller data (e.g., temperature, pressure, flow rate, etc.), can be derived for monitoring, control, and maintenance tasks as well for fail-safe control. Results are shared with process control systems via conventional 4-20 mA connections (for compatibility to current automation landscapes) as well as an integrated OPC-UA server over intrinsically safe Ethernet cable or Wi-Fi connection.
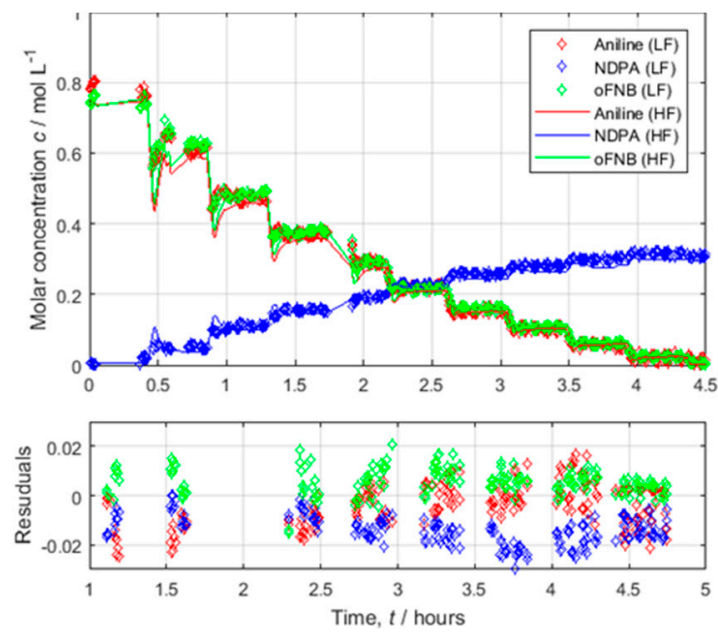

(a)

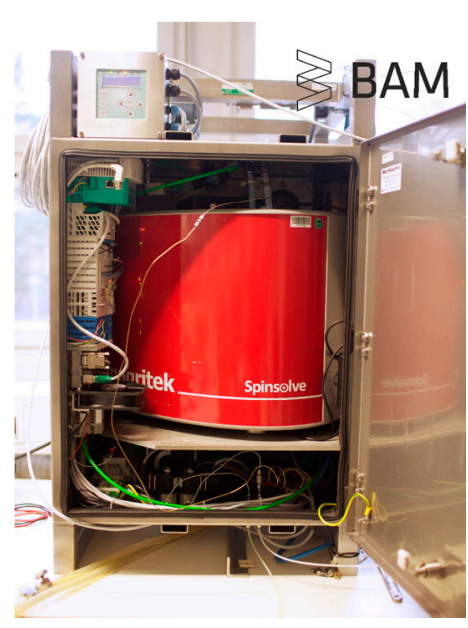

(b)

Figure 2. Smart NMR sensor module: (a) Monitoring of the aromatic coupling of 1-Fluoro-2nitrobenzene (FNB) with aniline to 2-Nitrodiphenylamine (NDPA) using Lithium-bis(trimethylsilyl) amide (Li-HMDS) in semi batch operation. Online 43.5 MHz low-field NMR (LF) was compared to $500 \mathrm{MHz}$ high-field NMR spectroscopy (HF) as reference method. Mole fractions are plotted versus time, observed deviations are below $20 \mathrm{mmol} / \mathrm{L}$ over the whole working range; (b) $43.5 \mathrm{MHz}$ benchtop online NMR sensor in an explosion proof housing (EX II 2G Ex pxb db ib IIB T3 Gb) of $57 \times 57 \times 85 \mathrm{~cm}$ module size.

Acknowledgments: Funding of CONSENS [3] by the European Union's Horizon 2020 research and innovation programme under grant agreement $\mathrm{N}^{\circ} 636942$ is gratefully acknowledged.

Conflicts of Interest: The authors declare no conflict of interest.

\section{References}

1. Meyer, K.; Kern, S.; Zientek, N.; Guthausen, G.; Maiwald, M. Process control with compact NMR. Trends Anal. Chem. 2016, 83, 39-52.

2. Lee, E.A. Cyber Physical Systems: Design Challenges; EECS Dep., University of California: Berkeley, CA, USA, 2008. Available online: http://www2.eecs.berkeley.edu/Pubs/TechRpts/2008/EECS-2008-8.pdf (accessed on 23 June 2017).

3. Technologie-Roadmap 4.0, Published by VDI/VDE Society Measurement and Automatic Control (VDI/VDE-GMA) and NAMUR-User Association of Automation Technology in Process Industries, Düsseldorf, Germany, 2015. Available online: http://www.namur.net/ (accessed 23 June 2017).

4. European Union's Horizon 2020 Project CONSENS-Integrated Control and Sensing for Sustainable Operation of Flexible Intensified Processes. Available online: www.consens-spire.eu (accessed on 23 June 2017).

(C) 2017 by the authors. Licensee MDPI, Basel, Switzerland. This article is an open access article distributed under the terms and conditions of the Creative Commons Attribution (CC BY) license (http://creativecommons.org/licenses/by/4.0/). 